\title{
AN APPLICATION OF PLEMELJ-SMITHIES FORMULAS TO COMPUTING GENERALIZED INVERSES OF FREDHOLM OPERATORS
}

\author{
Grażyna Ciecierska \\ Faculty of Mathematics and Computer Science, University of Warmia and Mazury in Olsztyn \\ Olsztyn, Poland \\ grac@matman.uwm.edu.pl
}

\begin{abstract}
We consider continuous operators $S+T$ in Banach spaces, where $S$ is Fredholm and $T$ is quasinuclear. By referring to the basic result of the Fredholm theory, i.e. to the expression of the resolvent $(I+\lambda T)^{-1}$ of the operator $T$ as a quotient of entire functions of $\lambda$, we derive analogous formulas for generalized inverses of operators $S+T$. We apply the Plemelj-Smithies formulas describing terms of determinant systems for the quasinuclear perturbations of Fredholm operators.
\end{abstract}

Keywords: Fredholm theory, determinant system, quasinuclear operator, generalized inverse

\section{Introduction}

The classical Fredholm theory [1] is concerned with the problem of solving the equation $(I+\lambda T) x=x_{0}$ in the space $C[a, b]$, where $T$ is an integral operator with the kernel continuous on $[a, b] \times[a, b]$. The theory has been extended by many authors, first by Riesz, Hilbert and Carleman, to square-integrable kernels. Grothendieck [2] and Ruston [3] have generalized the theory to nuclear operators in Banach spaces, Leżański [4], Sikorski [5-7] and Buraczewski [8, 9] to quasinuclear operators in Banach spaces. Later papers of Pietsch $[10,11]$ and König [12] dealt with absolutely 2 -summing operators and absolutely $p$-summing operators $(p>2)$ in Banach spaces, respectively. The further important contributions were made by Gohberg, Goldberg and Krupnik [13-15] for operators acting in Banach spaces and belonging to normed algebras with the approximation property.

The analytic formulas for determinants and subdeterminants for endomorphisms $I+T$ of $C[a, b]$, where $T$ is integral, were first given by Plemelj [16] in 1904 . A modification of these formulas was made by Carleman [17] and Smithies [18, 19] to make them applicable to endomorphisms $T$ belonging to the Hilbert- 
Schmidt class of a separable Hilbert space. The later results were obtained by Sikorski and Buraczewski in arbitrary Banach spaces. Sikorski [7] derived Plemelj-Smithies formulas for Fredholm endomorphisms $I+T, T$ being quasinuclear. Buraczewski [8] generalized these formulas in the case of endomorphisms $S+T$, where $S$ is any fixed Fredholm endomorphism of order zero with its given determinant system and $T$ is any quasinuclear endomorphism. The further generalization of the Plemelj-Smithies formulas in Banach spaces was made by Ciecierska [20] for operators $S+T, S$ being a fixed Fredholm operator with its given determinant system and $T$ being quasinuclear.

In the paper the Plemelj-Smithies formulas are used to provide generalized inverses of any quasinuclear perturbation of a fixed Fredholm operator acting in Banach spaces. By applying the results of the theory of determinant systems [7-9, 21 ], we derive formulas which generalize the basic result of the Fredholm theory, i.e. the expression of the resolvent $R(T ; \lambda)=(I+\lambda T)^{-1}$ of the operator $T$ as a quotient $\frac{D(T ; \lambda)}{d(T ; \lambda)}$ of entire functions of $\lambda \in C$. We refer to the result and express generalized inverses of any operator $S+T$ of order $r, S$ being a fixed Fredholm operator with its given determinant system $\left(D_{n}\right)_{n \in N \cup\{0\}}$ and $T$ being a quasinuclear operator determined by a quasinucleus $F$, as quotients $\frac{D_{r+1}(F)\left(\begin{array}{cccc}\xi, & \xi_{1}^{\prime}, & \ldots, & \xi_{r+d}^{\prime} \\ y, & y_{1}^{\prime}, \ldots, & y_{r}^{\prime}\end{array}\right)}{D_{r}(F)\left(\begin{array}{ccc}\xi_{1}^{\prime}, & \ldots, & \xi_{r+d}^{\prime} \\ y_{1}^{\prime}, & \ldots, & y_{r}^{\prime}\end{array}\right)}$. The functions $D_{r}(F)$ and $D_{r+1}(F)$ are entire functions, coefficients of which can be expressed by terms of a determinant system for $S+T$.

\section{Preliminaries}

We begin by recalling the terminology used in the theory of determinant systems. The notation is adopted from papers [5-9, 20,21].

Given two pairs $(\Xi, X),(\Omega, Y)$ of conjugate linear spaces over the same real or complex field $K$, we consider multilinear functionals on $\Xi^{\mu} \times Y^{m}, \mu, m \in N \cup\{0\}$. If $D$ is a $(\mu+m)$-linear functional on $\Xi^{\mu} \times Y^{m}$, then $D\left(\begin{array}{ll}\xi_{1}, \ldots, & \xi_{\mu} \\ y_{1}, \ldots, & y_{m}\end{array}\right)$ denotes its value at a point $\left(\xi_{1}, \ldots, \quad \xi_{\mu}, y_{1}, \ldots, \quad y_{m}\right) \in \Xi^{\mu} \times Y^{m}$. In the case $\mu=m=1$, i.e. if $D$ is a bilinear functional on $\Xi \times Y$, $\xi D y$ denotes the value of $D$ at a point $(\xi, y) \in \Xi \times Y$. A $(\mu+m)$-linear functional $D$ on $\Xi^{\mu} \times Y^{m}$ is called bi-skew symmetric if it is skew symmetric both in variables $\xi_{1}, \ldots, \xi_{\mu}$ and $y_{1}, \ldots, y_{m}$, i.e. for any 
permutations $\mathrm{p}=\left(p_{1}, \ldots, p_{\mu}\right), \mathrm{q}=\left(q_{1}, \ldots, q_{m}\right)$ of numbers $1, \ldots, \mu$ and $1, \ldots, m$, respectively, the following identity holds:

$$
D\left(\begin{array}{lll}
\xi_{p_{1}}, \ldots, & \xi_{p_{\mu}} \\
y_{q_{1}}, \ldots, & y_{q_{m}}
\end{array}\right)=\operatorname{sgn} \mathrm{p} \operatorname{sgn} \mathrm{q} D\left(\begin{array}{lll}
\xi_{1}, & \ldots, & \xi_{\mu} \\
y_{1}, & \ldots, & y_{m}
\end{array}\right) .
$$

We denote by $b s s_{\mu, m}(\Xi, Y)$ the set of all bi-skew symmetric functionals on $\Xi^{\mu} \times Y^{m} . D$ is called $(\Omega, X)$ - functional on $\Xi^{\mu} \times Y^{m}$ if for arbitrary fixed elements $\xi_{1}, \ldots, \xi_{i-1}, \xi_{i+1}, \ldots, \xi_{\mu} \in \Xi$ and $y_{1}, \ldots, y_{m} \in \mathrm{Y}$ there exists an element $x_{i} \in X$ such that $\xi x_{i}=D\left(\begin{array}{ccc}\xi_{1}, \ldots, & \xi_{i-1}, \xi, \xi_{i+1}, \ldots, & \xi_{\mu} \\ y_{1}, & \ldots, & y_{m}\end{array}\right)$ for every $\xi \in \Xi(i=1, \ldots, \mu)$ and for arbitrary fixed elements $\xi_{1}, \ldots, \xi_{\mu} \in \Xi, y_{1}, \ldots, y_{j-1}, y_{j+1}, \ldots, y_{m} \in Y$ there exists an element $\omega_{j} \in \Omega$ such that $\omega_{j} y=D\left(\begin{array}{ccc}\xi_{1}, & \ldots, & \xi_{\mu} \\ y_{1}, \ldots, & y_{j-1}, y, y_{j+1}, \ldots, & y_{m}\end{array}\right)$ for every $y \in Y \quad(j=1, \ldots, m)$. A bilinear $(\Omega, X)$ - functional on $\Xi \times Y$ is said to be an operator on $\Xi \times Y$. We use $L_{\mu, m}(\Xi, Y)$ to denote the set of all $(\Omega, X)$ - functionals on $\Xi^{\mu} \times Y^{m}$; in the case $\mu=m=1, o p(\Xi \rightarrow \Omega, Y \rightarrow X)$ denotes the set of all operators on $\Xi \times Y$. Each functional $D \in o p(\Xi \rightarrow \Omega, Y \rightarrow X)$ can be interpreted both as a linear mapping $D: \Xi \rightarrow \Omega$ and as a linear mapping $D: Y \rightarrow X$. Thus $\xi D y=(\xi D) y=\xi(D y)$ for $(\xi, y) \in \Xi \times Y$. For fixed non-zero elements $x_{0} \in X$, $\omega_{0} \in \Omega$, the operator $x_{0} \cdot \omega_{0}$ defined by $\xi\left(x_{0} \cdot \omega_{0}\right) y=\xi x_{0} \cdot \omega_{0} y$ for $(\xi, y) \in \Xi \times Y$ is called a one-dimensional operator on $\Xi \times Y$.

Given $A \in o p(\Omega \rightarrow \Xi, X \rightarrow Y)$, we define $N(A)=\{x \in X: A x=0\}$, $R(A)=\{A x: x \in X\}, \mathcal{N}(A)=\{\omega \in \Omega: \omega A=0\}, \mathcal{R}(A)=\{\omega A: \omega \in \Omega\} . A$ is said to be a Fredholm operator on $\Omega \times X$ of order $r(A)=\min \left\{n^{\prime}, m^{\prime}\right\}$ and index $d(A)=n^{\prime}-m^{\prime}, \quad$ if $\quad \operatorname{dim} N(A)=n^{\prime}<\infty, \quad \operatorname{dim} \mathcal{N}(A)=m^{\prime}<\infty, \quad R(A)=\mathcal{N}(A)^{\perp}$ and $\mathcal{R}(A)=N(A)^{\perp}$. An operator $B \in o p(\Xi \rightarrow \Omega, Y \rightarrow X)$ such that $A B A=A$, $B A B=B$ is called a generalized inverse of $A$.

A sequence $\left(D_{n}\right)_{n \in N \cup\{0\}}$ fulfilling the conditions:

(d3) there exists $r \in N \cup\{0\}$ such that $D_{r} \neq 0$;

(d4) the following identities hold for $n \in N \cup\{0\}$ : 


$$
\begin{gathered}
D_{n+1}\left(\begin{array}{ccc}
\xi_{0}, & \ldots, & \xi_{\mu_{n}} \\
A x, & y_{1}, \ldots, & y_{m_{n}}
\end{array}\right)=\sum_{i=0}^{\mu_{n}}(-1)^{i} \xi_{i} x \cdot D_{n}\left(\begin{array}{ccc}
\xi_{0}, \ldots, & \xi_{i-1}, \xi_{i+1}, \ldots, & \xi_{\mu_{n}} \\
y_{1}, & \ldots, & y_{m_{n}}
\end{array}\right), \\
D_{n+1}\left(\begin{array}{ccc}
\omega A, & \xi_{1}, \ldots, & \xi_{\mu_{n}} \\
y_{0}, & \ldots, & y_{m_{n}}
\end{array}\right)
\end{gathered}
$$

where $x \in X, \omega \in \Omega, \quad \xi_{i} \in \Xi, y_{j} \in Y, \quad i=1, \ldots, \mu_{n}, j=1, \ldots, m_{n}$, is said to be a determinant system for operator $A \in o p(\Omega \rightarrow \Xi, X \rightarrow Y)$. The least $r \in N \cup\{0\}$, such that $D_{r}$ does not vanish identically, and the difference $\mu_{0}-m_{0}$ are called the order and the index of the determinant system $\left(D_{n}\right)_{n \in N \cup\{0\}}$, respectively.

A linear functional $F$ on $o p(\Xi \rightarrow \Omega, Y \rightarrow X)$ is said to be a quasinucleus on $o p(\Xi \rightarrow \Omega, Y \rightarrow X)$, if there exists $T_{F} \in o p(\Omega \rightarrow \Xi, X \rightarrow Y)$ such that $F(x \cdot \omega)=\omega T_{F} x$ for $(\omega, x) \in \Omega \times X$. The set of all quasinuclei on $o p(\Xi \rightarrow \Omega, Y \rightarrow X)$ is denoted by $a n(\Omega \rightarrow \Xi, X \rightarrow Y) . T \in o p(\Omega \rightarrow \Xi, X \rightarrow Y)$ is called a quasinuclear operator on $\Omega \times X$, if there exists a quasinucleus $F \in \operatorname{an}(\Omega \rightarrow \Xi, X \rightarrow Y)$ such that $T=T_{F}$. The number $\operatorname{Tr} F=F(I)$ is said to be the trace of $F \in a n(\Xi \rightarrow \Xi, X \rightarrow X)$. For $F \in a n(\Omega \rightarrow \Xi, X \rightarrow Y)$ and $C \in o p(\Xi \rightarrow \Omega, Y \rightarrow X)$ we define $C F \in$ an $(\Xi \rightarrow \Xi, X \rightarrow X)$ :

$$
(C F)(A)=F(A C) \text { for } A \in o p(\Xi \rightarrow \Xi, X \rightarrow X) .
$$

Suppose $D \in b s s_{\mu, m}(\Xi, Y) \cap L_{\mu, m}(\Xi, Y)$ and $F \in a n(\Omega \rightarrow \Xi, X \rightarrow Y)$. We fix all the variables $\xi_{2}, \ldots \xi_{\mu} \in \Xi, y_{2}, \ldots, y_{m} \in Y$ and consider $D\left(\begin{array}{ll}\xi_{1}, \ldots, & \xi_{\mu} \\ y_{1}, \ldots, & y_{m}\end{array}\right)$ as the function of variables $\xi_{1}, y_{1}$ only, i.e. as the operator from op $(\Xi \rightarrow \Omega, Y \rightarrow X)$. The value of $F$ at the operator is denoted by $F \square D\left(\begin{array}{ll}\xi_{2}, \ldots, & \xi_{\mu} \\ y_{2} \ldots, & y_{m}\end{array}\right)$. Denote by $F \square D$ the function which assigns to $\xi_{2}, \ldots \xi_{\mu} \in \Xi, y_{2}, \ldots, y_{m} \in Y$ the number $F \square D\left(\begin{array}{cc}\xi_{2}, \ldots, & \xi_{\mu} \\ y_{2} \ldots, & y_{m}\end{array}\right)$. Thus $F \square D \in b s s_{\mu-1, m-1}(\Xi, Y)$. Assume, for $\mu>1, m>1$, that $F \square D \in L_{\mu-1, m-1}(\Xi, Y)$ and then repeat the above procedure to define $F \square F \square D \in b s s_{\mu-2, m-2}(\Xi, Y)$. We iterate the procedure $k$ - times, $k=\min \{\mu, m\}$, and define 


$$
F \square D, F \square F \square D, \ldots, \underbrace{F \square \ldots F}_{k-\text { times }} D,
$$

provided $\underbrace{F \square \ldots F \square}_{i \text {-times }} D \in L_{\mu-i, m-i}(\Xi, Y)(i \leq k)$. For a fixed $F \in a n(\Omega \rightarrow \Xi, X \rightarrow Y)$, $F \square$ denotes the function which assigns $F \square D$ to every $D \in b s s_{\mu, m}(\Xi, Y) \cap L_{\mu, m}(\Xi, Y)$. We also use the symbol $F^{\square k}$ to denote the modified $k$-th power of $F$, i.e. $F^{\mathbf{\square} k}=\frac{1}{k !} \underbrace{F \mathbf{a} \ldots \text { 口. }}_{k \text {-times }}$.

\section{Plemelj-Smithies formulas}

In this section we quote the basic results concerning the Plemelj-Smithies formulas, which are necessary for the proof of the main theorem of the paper.

Consider a continuous kernel $K:[a, b] \times[a, b] \rightarrow R$ and the induced Fredholm operator

$$
T x(t)=\int_{a}^{b} K(t, s) x(s) d s,
$$

$T: C[a, b] \rightarrow C[a, b]$. In the classical Fredholm determinant theory the equation

$$
(I+\lambda T) x=x_{0}
$$

has a unique solution

$$
x=R(T ; \lambda) x_{0},
$$

where $R(T ; \lambda)$ is the resolvent of the operator $T$, i.e. $R(T ; \lambda)=(I+\lambda T)^{-1}$, provided $-\frac{1}{\lambda}$ is not an eigenvalue of $T$. The basic result of the theory is to write the resolvent operator $R(T ; \lambda)$ as a quotient

$$
R(T ; \lambda)=\frac{D(T ; \lambda)}{d(T ; \lambda)}
$$

of entire functions of $\lambda \in C$. Plemelj [16] wrote explicitly $d$ and $D$ in (1) in terms of their power series

$$
d(T ; \lambda)=\sum_{k=0}^{\infty} \frac{\lambda^{k}}{k !} d_{k}(T)
$$




$$
D(T ; \lambda)=\sum_{k=0}^{\infty} \frac{\lambda^{k}}{k !} D_{k}(T),
$$

where $d_{k}$ are complex valued coefficients and $D_{k}$ are operator valued coefficients:

$$
d_{k}(T)=\left|\begin{array}{cccccc}
\operatorname{tr}(T) & k-1 & 0 & \ldots & 0 & 0 \\
\operatorname{tr}\left(T^{2}\right) & \operatorname{tr}(T) & k-2 & \ldots & 0 & 0 \\
\vdots & \vdots & \vdots & & \vdots & \vdots \\
\operatorname{tr}\left(T^{k-1}\right) & \operatorname{tr}\left(T^{k-2}\right) & \operatorname{tr}\left(T^{k-3}\right) & \ldots & \operatorname{tr}(T) & 1 \\
\operatorname{tr}\left(T^{k}\right) & \operatorname{tr}\left(T^{k-1}\right) & \operatorname{tr}\left(T^{k-2}\right) & \ldots & \operatorname{tr}\left(T^{2}\right) & \operatorname{tr}(T)
\end{array}\right|
$$

and

$$
D_{k}(T)=\left|\begin{array}{cccccc}
T^{0} & k & 0 & \ldots & 0 & 0 \\
T^{1} & \operatorname{tr}(T) & k-1 & \ldots & 0 & 0 \\
\vdots & \vdots & \vdots & & \vdots & \vdots \\
T^{k-1} & \operatorname{tr}\left(T^{k-1}\right) & \operatorname{tr}\left(T^{k-2}\right) & \ldots & \operatorname{tr}(T) & 1 \\
T^{k} & \operatorname{tr}\left(T^{k}\right) & \operatorname{tr}\left(T^{k-1}\right) & \ldots & \operatorname{tr}\left(T^{2}\right) & \operatorname{tr}(T)
\end{array}\right|
$$

with $T^{0}=I$.

Sikorski [7] generalized previous expressions for quantities $d(T)$ and $D(T)$, due to Grothendieck, Fredholm and Plemelj, over arbitrary Banach spaces. He presented expressions both for the determinant and subdeterminants of any order of the endomorphism $I+T$. Let $\left(\Theta_{n}\right)_{n \in N \cup\{0\}}$ be a determinant system for $I \in o p(\Xi \rightarrow \Xi, X \rightarrow X)$ and let $T \in o p(\Xi \rightarrow \Xi, X \rightarrow X)$ be a quasinuclear endomorphism determined by a quasinucleus $F \in a n(\Xi \rightarrow \Xi, X \rightarrow X)$, i.e. $T=T_{F}$. We recall that:

$$
\Theta_{n, k}(F)=F^{\mathbf{\square} k} \Theta_{n+k}, \quad \text { for } n, k \in N \cup\{0\}
$$

and that $\left(\Theta_{n}(F)\right)_{n \in N \cup\{0\}}$ defined by

$$
\Theta_{n}(F)=\sum_{k=0}^{\infty} \Theta_{n, k}(F)
$$

is a determinant system for $I+T \in o p(\Xi \rightarrow \Xi, X \rightarrow X)$. Sikorski expressed the coefficients of the analytic functions (7) by determinants involving traces of 
iterates of $T$. More precisely, let $T_{n}^{k}$ denote the $2 n$-linear functional defined on $\Xi^{n} \times X^{n}$ by the formula

$$
T_{n}^{k}\left(\begin{array}{ll}
\xi_{1}, \ldots, & \xi_{n} \\
x_{1}, \ldots, & x_{n}
\end{array}\right)=\sum\left|\begin{array}{ccc}
\xi_{1} T^{i_{1}} x_{1} & \ldots & \xi_{1} T^{i_{1}} x_{n} \\
\vdots & & \vdots \\
\xi_{n} T^{i_{n}} x_{1} & \ldots & \xi_{n} T^{i_{n}} x_{n}
\end{array}\right|,
$$

where the summation is extended over all finite sequences of non-negative integers $i_{1}, \ldots, i_{n}$ such that $i_{1}+\ldots+i_{n}=k$ for $k \in N \cup\{0\}$ and $n \in N$. The Plemelj-Smithies formulas for $\Theta_{n, k}(F)$ in (6) are of the form

$$
\Theta_{0,0}(F)=1, \quad \Theta_{0, k}(F)=\frac{1}{k !}\left|\begin{array}{cccccc}
\sigma_{1} & k-1 & 0 & \ldots & 0 & 0 \\
\sigma_{2} & \sigma_{1} & k-2 & \ldots & 0 & 0 \\
\vdots & \vdots & \vdots & & \vdots & \vdots \\
\sigma_{k-1} & \sigma_{k-2} & \sigma_{k-3} & \ldots & \sigma_{1} & 1 \\
\sigma_{k} & \sigma_{k-1} & \sigma_{k-2} & \ldots & \sigma_{2} & \sigma_{1}
\end{array}\right|, k \in N
$$

and

$$
\Theta_{n, k}(F)=\frac{1}{k !}\left|\begin{array}{cccccc}
T_{n}^{0} & k & 0 & \ldots & 0 & 0 \\
T_{n}^{1} & \sigma_{1} & k-1 & \ldots & 0 & 0 \\
\vdots & \vdots & \vdots & & \vdots & \vdots \\
T_{n}^{k-1} & \sigma_{k-1} & \sigma_{k-2} & \ldots & \sigma_{1} & 1 \\
T_{n}^{k} & \sigma_{k} & \sigma_{k-1} & \ldots & \sigma_{2} & \sigma_{1}
\end{array}\right|, n \in N, k \in N \cup\{0\},
$$

where

$$
\sigma_{k}=F\left(T^{k-1}\right)=\operatorname{Tr}\left(T^{k}\right) \text { for } k \in N .
$$

Buraczewski [8] extended Sikorski's result to endomorphisms $S+T$, where $S$ is Fredholm of order zero and $T$ is quasinuclear. Let $\left(D_{n}\right)_{n \in N \cup\{0\}}$ be a determinant system for $S \in o p(\Xi \rightarrow \Xi, X \rightarrow X)$ of order $r(S)=0$ and index $d(S)=d \geq 0$, $U \in o p(\Xi \rightarrow \Xi, X \rightarrow X)$ be a generalized inverse of $S,\left\{z_{1}, \ldots, z_{d}\right\}$ be a complete system of solutions of the homogenous equation $S x=0$. For a quasinucleus $F \in \operatorname{an}(\Xi \rightarrow \Xi, X \rightarrow X)$, which determines the quasinuclear operator $T=T_{F} \in o p(\Xi \rightarrow \Xi, X \rightarrow X)$ let

$$
D_{n, k}(F)=F^{\square k} D_{n+k}, \quad n, k \in N \cup\{0\} .
$$


Then the sequence $\left(D_{n}(F)\right)_{n \in N \cup\{0\}}$ defined by

$$
D_{n}(F)=\sum_{k=0}^{\infty} D_{n, k}(F)
$$

is a determinant system for $S+T$ and the Plemelj-Smithies formulas are of the analogous form as (8) and (9), where $T_{n}^{k}$ denotes the $(2 n+d)$-linear functional defined on $\Xi^{n+d} \times X^{n}$ by the formula

$$
\begin{gathered}
T_{n}^{k}\left(\begin{array}{ccc}
\xi_{1}, \ldots, & \xi_{n+d} \\
x_{1}, \ldots, & x_{n}
\end{array}\right)= \\
=\sum\left|\begin{array}{cccccc}
\xi_{1}(U T)^{i_{1}} U x_{1} & \ldots & \xi_{1}(U T)^{i_{1}} U x_{n} & \xi_{1}(U T)^{i_{1}} z_{1} & \ldots & \xi_{1}(U T)^{i_{1}} z_{d} \\
\vdots & \vdots & \vdots & & \vdots \\
\xi_{n+d}(U T)^{i_{n+d}} U x_{1} & \ldots & \xi_{n+d}(U T)^{i_{n+d}} U x_{n} & \xi_{n+d}(U T)^{i_{n+d}} z_{1} & \ldots & \xi_{1}(U T)^{i_{n+d}} z_{d}
\end{array}\right|,
\end{gathered}
$$

the summation being extended over all finite sequences of non-negative integers $i_{1}, \ldots, i_{n+d}$ such that $i_{1}+\ldots+i_{n+d}=k$ and

$$
\sigma_{k}=F\left((U T)^{k-1} U\right)=\operatorname{Tr}\left((U T)^{k}\right) \text { for } k \in N .
$$

Ciecierska [20] obtained the further generalization of the above result and derived the Plemelj-Smithies formulas for any operator $S+T$, $S \in o p(\Omega \rightarrow \Xi, X \rightarrow Y)$ being Fredholm and $T \in o p(\Omega \rightarrow \Xi, X \rightarrow Y)$ being quasinuclear. Let $F \in a n(\Omega \rightarrow \Xi, X \rightarrow Y)$ determine $T$ and $\left\{z_{1}, \ldots, z_{n^{\prime}}\right\}$, $\left\{\varsigma_{1}, \ldots, \varsigma_{m^{\prime}}\right\}$ be complete systems of solutions of the homogenous equations $S x=0$ and $\omega S=0$, respectively. For a determinant system $\left(D_{n}\right)_{n \in N \cup\{0\}}$ for $S$ and a generalized inverse $U \in \operatorname{op}(\Xi \rightarrow \Omega, Y \rightarrow X)$ of $S$, the following formulas hold:

$$
\underbrace{\text { ㅁ.. } \mathbf{a} \mathbf{q}}_{k-\text { times }} D_{n+k}=\left|\begin{array}{cccccc}
T_{n}^{0} & k & 0 & \ldots & 0 & 0 \\
T_{n}^{1} & \sigma_{1} & k-1 & \ldots & 0 & 0 \\
\vdots & \vdots & \vdots & & \vdots & \vdots \\
T_{n}^{k-1} & \sigma_{k-1} & \sigma_{k-2} & \ldots & \sigma_{1} & 1 \\
T_{n}^{k} & \sigma_{k} & \sigma_{k-1} & \ldots & \sigma_{2} & \sigma_{1}
\end{array}\right|
$$

for $n, k \in N \cup\{0\}$, where

$$
\sigma_{k}=\operatorname{Tr}\left((U T)^{k-1} U F\right)
$$


$T_{n}^{0}=D_{n}$ and $T_{n}^{k}$ denotes the $\left(2 n+n^{\prime}+m^{\prime}-2 r\right)$-linear functional defined on $\Xi^{n+n^{\prime}-r} \times X^{n+m^{\prime}-r}$ by the formula

$$
\begin{aligned}
& T_{n}^{k}\left(\begin{array}{ll}
\xi_{1}, \ldots, & \xi_{n+n^{\prime}-r} \\
y_{1}, \ldots, & y_{n+m^{\prime}-r}
\end{array}\right)=
\end{aligned}
$$

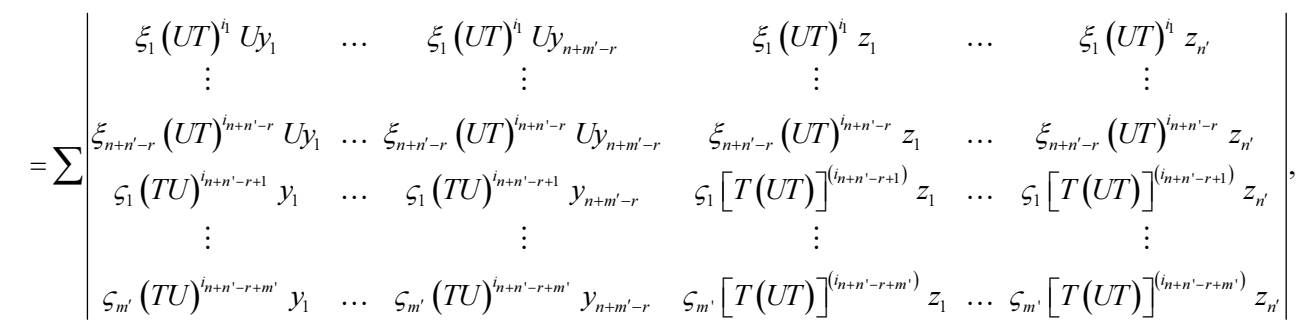

where for $s=1, \ldots m^{\prime}, t=1, \ldots n^{\prime}$

$$
\varsigma_{s}[T(U T)]^{\left(i_{n+n^{\prime}-r+s}\right)} z_{t}=\left\{\begin{array}{clc}
0 & \text { if } & i_{n+n^{\prime}-r+s}=0 \\
\varsigma_{s} T(U T)^{i_{n+n^{\prime}-r+s}-1} z_{t} & \text { if } & i_{n+n^{\prime}-r+s}=1, \ldots k
\end{array}\right.
$$

and $\sum$ is extended over all finite sequences of non-negative integers $i_{1}, \ldots i_{n+n^{\prime}-r+m^{\prime}}$, such that $i_{1}+\ldots+i_{n+n^{\prime}-r+m^{\prime}}=k$.

\section{The main result}

In this section we describe generalized inverses of a quasinuclear perturbation of a fixed Fredholm operator acting in Banach spaces. As a tool of the description we use terms of a determinant system for the mentioned perturbation. We begin with the following consequence of the main result of [20].

Lemma 3.1. If $\left(D_{n}\right)_{n \in N \cup\{0\}}$ is a determinant system for a Fredholm operator $S \in o p(\Omega \rightarrow \Xi, X \rightarrow Y)$ of order $r=\min \left\{n^{\prime}, m^{\prime}\right\}$ and index $d=n^{\prime}-m^{\prime} \geq 0$, then for any $F \in \operatorname{an}(\Omega \rightarrow \Xi, X \rightarrow Y)$ the sequence $\left(D_{n}(F)\right)_{n \in N \cup\{0\}}$ :

$$
D_{n}(F)=\sum_{k=0}^{\infty} D_{n, k}(F),
$$

where

$$
D_{0,0}(F)=1, \quad D_{0, k}(F)=\frac{1}{k !}\left|\begin{array}{cccccc}
\sigma_{1} & k-1 & 0 & \ldots & 0 & 0 \\
\sigma_{2} & \sigma_{1} & k-2 & \ldots & 0 & 0 \\
\vdots & \vdots & \vdots & & \vdots & \vdots \\
\sigma_{k-1} & \sigma_{k-2} & \sigma_{k-3} & \ldots & \sigma_{1} & 1 \\
\sigma_{k} & \sigma_{k-1} & \sigma_{k-2} & \ldots & \sigma_{2} & \sigma_{1}
\end{array}\right|, \quad k \in N
$$


and

$$
D_{n, k}(F)=\frac{1}{k !}\left|\begin{array}{cccccc}
T_{n}^{0} & k & 0 & \ldots & 0 & 0 \\
T_{n}^{1} & \sigma_{1} & k-1 & \ldots & 0 & 0 \\
\vdots & \vdots & \vdots & & \vdots & \vdots \\
T_{n}^{k-1} & \sigma_{k-1} & \sigma_{k-2} & \ldots & \sigma_{1} & 1 \\
T_{n}^{k} & \sigma_{k} & \sigma_{k-1} & \ldots & \sigma_{2} & \sigma_{1}
\end{array}\right|, \quad n \in N, k \in N \cup\{0\}
$$

$\sigma_{k}$ being defined by (16) and $T_{n}^{k}$ being defined by (17), is a determinant system for $S+T_{F}$.

Now we are in a position to describe a generalized inverse of a quasinuclear perturbation of a fixed Fredholm operator.

Theorem 3.2. We assume that:

(i) $S \in o p(\Omega \rightarrow \Xi, X \rightarrow Y)$ is a Fredholm operator of index $d \geq 0$ and of a determinant system $\left(D_{n}\right)_{n \in N \cup\{0\}}$;

(ii) $F \in \operatorname{an}(\Omega \rightarrow \Xi, X \rightarrow Y)$ determines the quasinuclear operator $T \in o p(\Omega \rightarrow \Xi, X \rightarrow Y)$

(iii) $S+T \in o p(\Omega \rightarrow \Xi, X \rightarrow Y)$ is of order $r(S+T)=r^{\prime}$ and the determinant system $\left(D_{n}(F)\right)_{n \in N \cup\{0\}}$ defined by (18)-(20);

(iv) $y_{1}^{\prime}, \ldots, y_{r^{\prime}}^{\prime} \in Y, \xi_{1}^{\prime}, \ldots, \xi_{r^{\prime}+d}^{\prime} \in \Xi$ are such that

$$
\delta=D_{r^{\prime}}(F)\left(\begin{array}{ccc}
\xi_{1}^{\prime}, & \ldots, & \xi_{r^{\prime}+d}^{\prime} \\
y_{1}^{\prime}, & \ldots, & y_{r^{\prime}}^{\prime}
\end{array}\right) \neq 0 .
$$

Then the operator $B \in o p(\Xi \rightarrow \Omega, Y \rightarrow X)$ defined by the formula

$$
\xi B y=\frac{D_{r^{\prime}+1}(F)\left(\begin{array}{lll}
\xi, & \xi_{1}^{\prime}, \ldots, & \xi_{r^{\prime}+d}^{\prime} \\
y, & y_{1}^{\prime}, \ldots, & y_{r^{\prime}}^{\prime}
\end{array}\right)}{D_{r^{\prime}}(F)\left(\begin{array}{ccc}
\xi_{1}^{\prime}, & \ldots, & \xi_{r^{\prime}+d}^{\prime} \\
y_{1}, & \ldots, & y_{r^{\prime}}
\end{array}\right)} \text { for }(\xi, y) \in \Xi \times Y,
$$

where coefficients of the analytic functions $D_{r^{\prime}}(F)$ and $D_{r^{\prime}+1}(F)$ are expressed by Plemelj-Smithies formulas, is a generalized inverse of $S+T$. 
Proof. Since $\left(D_{n}(F)\right)_{n \in N \cup\{0\}}$ is a determinant system for $S+T$ and $r(S+T)=r^{\prime}$, $D_{r^{\prime}}(F) \neq 0$. Moreover, elements $y_{1}^{\prime}, \ldots, y_{r^{\prime}}^{\prime} \in Y$ and $\xi_{1}^{\prime}, \ldots, \xi_{r^{\prime}+d}^{\prime} \in \Xi$ satisfy the condition (21). Since $D_{r^{\prime}}(F)$ is $(\Omega, X)$ - functional on $\Xi^{r^{\prime}+d} \times Y^{r^{\prime}}$, there are elements $\varsigma_{1}, \ldots, \varsigma_{r^{\prime}} \in \Omega$ such that

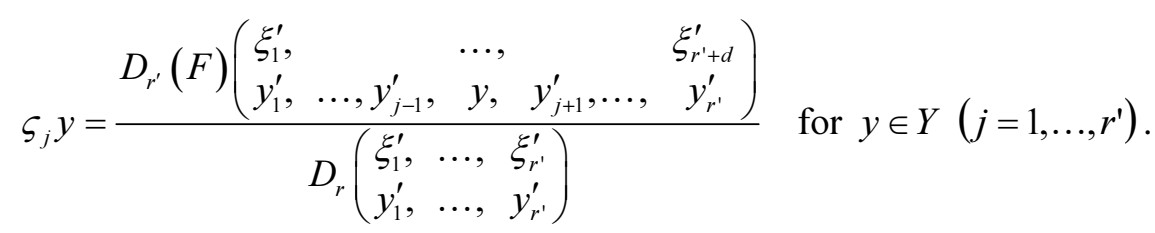

Furthermore, it follows from (23) and from a bi-skew symmetry of $D_{r^{\prime}}(F)$ that $\varsigma_{j} y^{\prime}=\delta_{i j}\left(i, j=1, \ldots, r^{\prime}\right)$. Thus $\varsigma_{1}, \ldots, \varsigma_{r^{\prime}}$ and $y_{1}^{\prime}, \ldots, y_{r^{\prime}}^{\prime}$ are linearly independent. Similarly, bearing in mind that $D_{r^{\prime}}(F) \in L_{r^{\prime}+d, r^{\prime}}(\Xi, Y)$, there are elements $z_{1}, \ldots, z_{r^{\prime}+d} \in X$ satisfying the condition

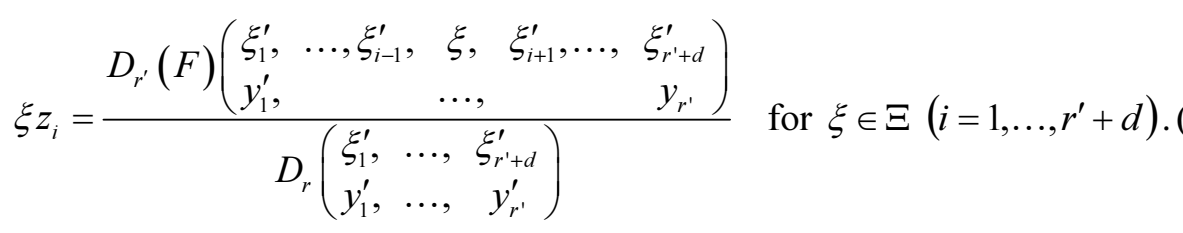

Since $D_{r^{\prime}}(F)$ is bi-skew symmetric, it follows from (24) that $\xi_{j}^{\prime} z_{i}=\delta_{i j}$ $\left(i, j=1, \ldots, r^{\prime}+d\right)$. Consequently, elements $z_{1}, \ldots, z_{r^{\prime}+d}$ and $\xi_{1}^{\prime}, \ldots, \xi_{r^{\prime}+d}^{\prime}$ are also linearly independent. Moreover, the elements $\varsigma_{1}, \ldots, \varsigma_{r^{\prime}}$ are solutions of the homogenous equation $\omega(S+T)=0$, because (23), (d4) and a bi-skew symmetry of $D_{r^{\prime}}(F)$ imply that

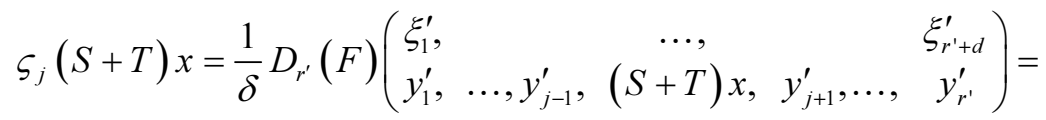

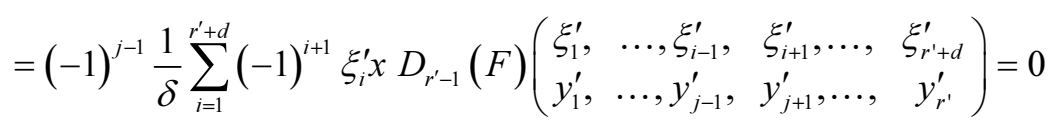

for each $x \in X$. Similarly, elements $z_{1}, \ldots, z_{r^{\prime}+d}$ are solutions of the homogenous equation $(S+T) x=0$. Indeed, since $D_{r^{\prime}}(F) \in b s s_{r^{\prime}+d, r^{\prime}}(\Xi, Y)$, in view of (24), (d4), we obtain

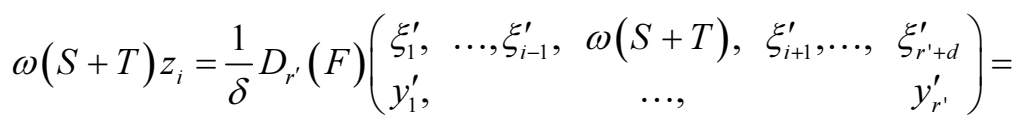




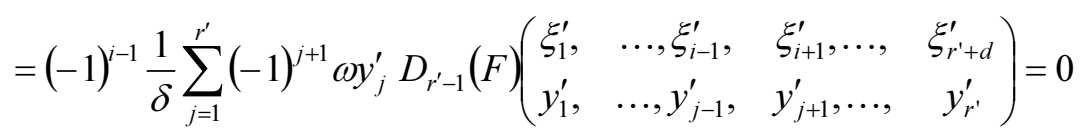

for each $\omega \in \Omega$. Thus, $\left\{z_{1}, \ldots, z_{r^{\prime}+d}\right\}$ and $\left\{\varsigma_{1}, \ldots, \varsigma_{r^{\prime}}\right\}$ are complete systems of solutions of the equations $(S+T) x=0$ and $\omega(S+T)=0$, respectively.

Replacing $\xi$ by $\omega(S+T)$ in (22) and then applying (d4) for $n=r^{\prime}$, in view of a bi-skew symmetry of $D_{r^{\prime}}(F)$, we obtain

$$
\begin{aligned}
& \omega(S+T) B y=\frac{1}{\delta} D_{r^{\prime}+1}(F)\left(\begin{array}{cccc}
\omega(S+T) & \xi_{1}^{\prime}, & \ldots, & \xi_{r^{\prime}+d}^{\prime} \\
y, & y_{1}^{\prime}, & \ldots, & y_{r^{\prime}}^{\prime}
\end{array}\right)=
\end{aligned}
$$

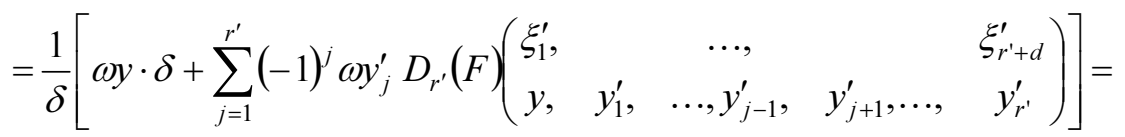

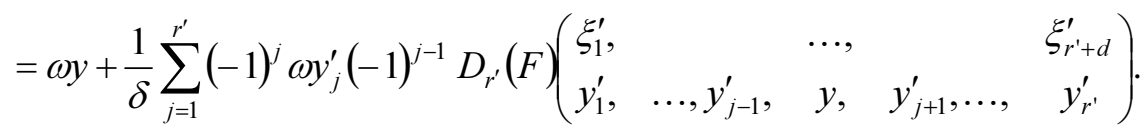

Taking into account (23), $\omega(S+T) B y=\omega y-\sum_{j=1}^{r^{\prime}} \omega y_{j}^{\prime} \cdot \varsigma_{j} y$ for each $(\omega, y) \in \Omega \times Y$. Hence,

$$
(S+T) B=J-\sum_{j=1}^{r^{\prime}} y_{j}^{\prime} \cdot \varsigma_{j},
$$

$J \in o p(\Omega \rightarrow \Omega, Y \rightarrow Y)$ being the identity operator. Analogously, replacing $y$ by $(S+T) x$ in (22) and then using (d4) for $n=r^{\prime}$, by a bi-skew symmetry of $D_{r^{\prime}}(F)$, we obtain

$$
\begin{aligned}
& \xi B(S+T) x=\frac{1}{\delta} D_{r^{\prime}+1}(F)\left(\begin{array}{cccc}
\xi & \xi_{1}^{\prime}, & \ldots, & \xi_{r^{\prime}+d}^{\prime} \\
(S+T) x, & y_{1}^{\prime}, & \ldots, & y_{r^{\prime}}^{\prime}
\end{array}\right)=
\end{aligned}
$$

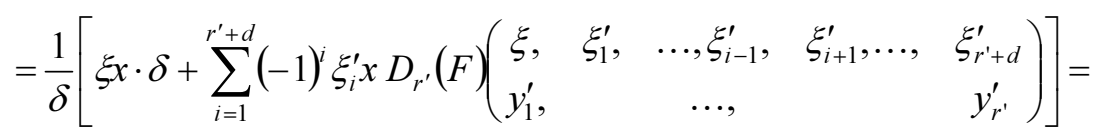

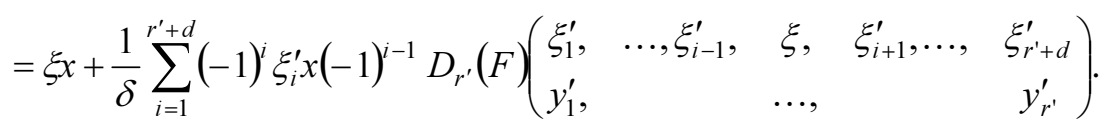


By (24), $\xi B(S+T) x=\xi x-\sum_{i=1}^{r^{\prime}+d} \xi z_{i} \cdot \xi_{i}^{\prime} x$ for each $(\xi, x) \in \Xi \times X$. Consequently,

$$
B(S+T)=I-\sum_{i=1}^{r^{\prime}+d} z_{i} \cdot \xi_{i}^{\prime}
$$

$I \in o p(\Xi \rightarrow \Xi, X \rightarrow X)$ being the identity operator. Formulas (25) and (26) imply that $(S+T) B(S+T)=S+T$ and $B(S+T) B=B$. This proves that $B$ is a generalized inverse of $S+T$. Now, Lemma 4.1. yields the required result.

\section{Conclusions}

In the paper quasinuclear perturbations of Fredholm operators in Banach spaces were considered. By applying the Plemelj-Smithies formulas, we described terms of determinant systems for the mentioned perturbations. Moreover, we expressed generalized inverses of such perturbations as quotients of entire functions. The obtained result, in the case of the invertible perturbation, leads to the well-known expression of the resolvent operator in the classical Fredholm theory.

\section{References}

[1] Fredholm I., Sur une classe d'equations fonctionnelles, Acta Math. 1903, 27, 365-390.

[2] Grothendieck A., La theorie de Fredholm, Bull. Soc. Math. Fr. 1956, 84, 319-384.

[3] Ruston A.F., On the Fredholm theory of integral equations for operators belonging to the trace class of a general Banach space, Proc. Lond. Math. Soc. III. Ser. 1951(2), 53, 109-124 .

[4] Leżański T., The Fredholm theory of linear equations in Banach spaces, Stud. Math. 1953, 13, 244-276.

[5] Sikorski R., On Leżański's determinants of linear equations in Banach spaces, Stud. Math. 1953, 14, 24-48.

[6] Sikorski R., Determinant systems, Stud. Math. 1959, 18, 161-186.

[7] Sikorski R., The determinant theory in Banach spaces, Colloq. Math. 1961, 8, 141-198.

[8] Buraczewski A., The determinant theory of generalized Fredholm operators, Studia Math. 1963, 22, 265-307.

[9] Buraczewski A., Sikorski R., Analytic formulae for determinant systems in Banach spaces, Studia Math. 1980, 67, 85-101.

[10] Pietsch A., Zur Fredholmschen Theorie in lokalkonvexen Räumen, Stud. Math. 1963, 22, 161-179.

[11] Pietsch A., Eigenvalues and s-Numbers, Cambridge Studies in Advanced Math., Vol. 13, Cambridge Univ. Press, Cambridge 1987.

[12] König H., A Fredholm determinant theory for $p$-summing maps in Banach spaces, Math. Ann. 1980, 247, 255-274. 
[13] Gohberg I., Goldberg S., Krupnik N., Traces and determinants of linear operators, Integr. Equat. Oper. Th. 1996, 26, 136-187.

[14] Gohberg I., Goldberg S., Krupnik N., Hilbert-Carleman and regularized determinants for linear operators, Integr. Equat. Oper. Th. 1997, 27, 10-47.

[15] Gohberg I., Goldberg S., Krupnik N., Generalization of the determinants for trace-potent linear operators, Integr. Equat. Oper. Th. 2001, 40, 441-453.

[16] Plemelj J., Zur Theorie der Fredholmschen Funktionalgleichung, Monat. Math. Phys. 1904, $15,93-128$.

[17] Carleman T., Zur Theorie der linearen Integralgleichungen, Math. Zeit. 1921, 9, 196-217.

[18] Smithies F., The Fredholm theory of integral equations, Duke Math. J. 1941, 107-130.

[19] Smithies F., Integral Equations, Cambridge Univ. Press, Cambridge 1970.

[20] Ciecierska G., On some application of algebraic quasinuclei to the determinant theory, Journal of Applied Mathematics and Computational Mechanics 2013, 12(3), 27-38.

[21] Ciecierska G., Determinant systems for nuclear perturbations of Fredholm operators in Frechet spaces, International Publications USA, Panam. Math. J. 2014, 24(1), 1-20. 